\title{
EVALUATION OF ANALGESIC EFFECT OF ACUPUNCTURE BY USING SEPS: AN OBJECTIVE STUDY
}

\author{
C. Satyasri1, C. Saroja², O. Padmini33, Kali Vara Prasad Vadlamani ${ }^{4}$
}

${ }_{1}^{1}$ Assistant Professor, Department of Physiology, Osmania Medical College, Hyderabad.

${ }^{2}$ Assistant Professor, Department of Physiology, Osmania Medical College, Hyderabad.

${ }^{3}$ Associate Professor, Department of Physiology, Osmania Medical College, Hyderabad.

${ }^{4}$ Associate Professor, Department of Orthopaedics, Osmania Medical College, Hyderabad.

\section{ABSTRACT}

\section{BACKGROUND}

Acupuncture is a form of alternative medicine, which originated in China in 100 BC. Special needles inserted into the body at special points were supposed to reduce pain sensation albeit subjectively. Since it modified the cognitive and affective sensations of pain, it was coupled with western treatment modalities. Evoked potentials are stand up waves, which are generated and conducted along sensory nerves on stimulation. Claims that these electrical impulses, especially in response to pain sense could be modified with acupuncture technique, were substantiated with several studies from time to time. Ours was one such attempt to provide objective evidence.

\section{MATERIAL AND METHODS}

The study was done in the Upgraded Department of Physiology, Osmania Medical College, Hyderabad, between December 2011 and December 2012; 21 subjects were chosen (11 males and 10 females) in the age group of 25-65 years suffering from pain in the upper limbs, neck and shoulders. A non-invasive method of estimation of nerve conduction using electromyography or evoked potential system using surface-electrodes with automated computerized monitor attached with printer was employed for studying pain related evoked potentials before and after application of the acupuncture technique on patients with upper limb pain of more than 3 weeks' duration.

\section{RESULTS AND CONCLUSION}

Suitable statistical analysis of pre-acupuncture and post-acupuncture data showed no significant difference in the latencies and inter-peak latencies, but a significant change in N9 and N20 wave amplitudes.

\section{CONCLUSION}

The results of our study suggest that the analgesic effect of Acupuncture does not modify the action potentials conducted along the neuronal pathways to any great extent. However, more light can be thrown on the subject by examining other nerves and studying a larger sample.

\section{KEYWORDS}

Acupuncture, Evoked Potentials, Pain Relief.

HOW TO CITE THIS ARTICLE: Satyasri C, Saroja C, Padmini O, et al. Evaluation of analgesic effect of acupuncture by using SEPs: an objective study. J. Evolution Med. Dent. Sci. 2016;5(70):5112-5115, DOI: 10.14260/jemds/2016/1159

\section{INTRODUCTION}

Acupuncture (From Latin, acus (Needle) and punctura (To puncture) is a form of alternative medicine and a key component of Traditional Chinese Medicine (TCM) involving thin needles inserted into the body at acupuncture points. ${ }^{1,2}$ Acupuncture is believed to have originated around $100 \mathrm{BC}$ in China around the time The Yellow Emperor's Classic of Internal Medicine (Huangdi Neijing) was published and singleuse needle. ${ }^{3}$ Over time, conflicting claims and belief systems emerged about the effect of lunar, celestial and earthly cycles, yin and yang energies, and a body's "rhythm" on the effectiveness of treatment. ${ }^{4}$ Acupuncture grew and diminished in popularity in China repeatedly, depending on the country's political leadership and the favour of rationalism or Western medicine.

Financial or Other, Competing Interest: None.

Submission 14-03-2016, Peer Review 20-04-2016,

Acceptance 26-04-2016, Published 01-09-2016.

Corresponding Author:

Dr. O. Padmini,

F 12, Sneha Enclave,

St. No. 4, West Maredpally,

Secunderabad,

Telangana.

E-mail: padiminiprasad91@gmail.com

DOI: $10.14260 /$ jemds/2016/1159
Acupuncture spread first to Korea in the 6th century AD, then to Japan through medical missionaries and then to Europe, starting with France. ${ }^{5}$ In the 20th century, as it spread to the United States and Western Countries, the spiritual elements of acupuncture that conflict with Western beliefs were abandoned in favour of tapping needles into nerves. 6,7

Acupuncture needling often evokes complex somatosensory sensations and may modulate the cognitive/affective perception of pain suggesting that many effects are supported by the brain and extending central nervous system networks.

Acupuncture is rarely used alone, but rather as an adjunct to other treatment modalities. Established guidelines recommend the use of acupuncture for the management of non-specific low back pain among other treatments. The American Society of Anaesthesiologists states it may be considered in the treatment for non-specific, noninflammatory low back pain only in conjunction with conventional therapy. ${ }^{8}$

Acupuncture is the stimulation of specific acupuncture points along the skin of the body using thin needles. It can be associated with the application of heat, pressure or laser light to these points. 
An Evoked Potential (EP) is an electrical manifestation of the brain's reception of and response to an external stimulus. EPs are electrical signals generated by the nervous system in response to sensory stimuli such as auditory, visual and somatosensory stimuli. The electrical signals evoked by somatosensory stimuli are called Somatosensory EPs (SEPs), and are used to evaluate the conduction of neural structures along the somatosensory pathways.

In the 1970s, SEP methodology started to be used in acupuncture studies. Pain-Related EPs (PREPs) and shortlatency SEPs (SLSEPs) are two kinds of SEPs that are commonly used in acupuncture studies. PREPs are evoked by pain stimuli. The later-latency components of PREPs $(>150$ $\mathrm{ms}$ ) are related to the sensation of pain. SLSEPs are conventional SEPs that are evoked by somatosensory stimuli, whose intensity is adjusted to induce a visible twitch of the related muscle, but no pain. SLSEPs are widely used in clinical practice, because they are rarely influenced by consciousness and have explicit neural origins.

Zeng et al found that electro-acupuncture on both Hegu (LI 4) and non-acupoints could inhibit the amplitudes of the medium and late components of PREPs, and the effect of electro-acupuncture on Hegu (LI 4) was strikingly superior to that of electro-acupuncture on non-acupoints. ${ }^{9}$ This study suggested that acupoints had a more noticeable effect of analgesia than non-acupoints. Acupoints ipsilateral to the nerve stimulation were more effective at reducing the amplitude of PREPs than those contralateral to the nerve on of PREPs and SLSEPs in clinical trials of acupuncture.

Our study was an attempt at objectively evaluating the analgesic efficacy of acupuncture by using EPs before and after acupuncture.

\section{MATERIAL AND METHODS}

The study was done in the Upgraded Department of Physiology, Osmania Medical College, Hyderabad. The study period extended between December 2011 and December 2012; 21 subjects were chosen (11 males and 10 females) in the age group of 25-65 years, suffering from pain in the upper limbs, neck and shoulders. Ethical guidelines were strictly followed and consent taken prior to study after the subjects were told about aim and objectives of the study.

The procedure was a non-invasive method of estimation of nerve conduction using SFEMG/EP- Electromyography or evoked potential system (Nicolet systems-USA make) using surface-electrodes with automated computerized monitor attached with printer. It was conducted on patients with pain in the upper limb, neck and shoulders of more than 3 weeks' duration using 3-channel normal averaging technique. The procedure was explained to the patient and consent taken. Patient was asked to sit comfortably on a chair and instructed to gently close his/her eyes while relaxing all the head and neck muscles during the recording. Patient was asked to count the number of stimuli so as to get proper recordings. Electrodes of the 3 channels were placed on the patient at appropriate sites after proper abrasion.

Surface EEG electrodes were used. Fz was used as the reference in all montages.

Single fiber electromyography (Nicolet system) was used for the recording of Evoked Potentials. Proper preparation of electrode-patient interface was done with mild abrasive and cleansing agent to remove skin oils, dirt and dead cells. Skin/scalp surface - cup electrodes with small central holes and cream were used. The area was then covered with cotton wool or adhesive tape amplification in reasonable steps, up to a gain factor of 5.00,000 X was applied. Analog-to-Digital Conversion (ADC) was mandatory, as ADC is an integral part of signal average. ADC capability is an important aspect of EP functionality. In EP studies, the wave form peak is most commonly used as the measurement point. The time separation between 2 peaks is termed 'inter-wave latency' or 'inter-peak latency' or absolute latency or implicit time.

Amplitude in EP work is Usually Stated in pV (Millionth of a volt). Active recording electrodes were placed as follows:

1. 1st channel-over the contralateral C37C4' scalp region ( $2 \mathrm{~cm}$ posterior to $\mathrm{C} 3$ or $\mathrm{C} 4$ )

2. 2nd channel-over the C5/C2 cervical spinous process (Referred to as $\mathrm{C} 5 \mathrm{~S}$ or $\mathrm{C} 2 \mathrm{~S}$ and located relative to the prominent $\mathrm{C} 7$ spinous process with the neck flexed.

3. 3rd channel-at Erb's point $(2-3 \mathrm{~cm}$ above clavicle in the angle between it and posterior border of the clavicular head of the Sternocleidomastoid muscle ipsilaterally). Inactive recording electrodes of the 3 channels were placed on Fz position on the scalp. Ground electrode was placed between the stimulating and the recording electrodes relatively close to the former. We have used a band around the forearm. Stimulation electrode using surface disk electrodes were placed between the tendons of palmaris longus and flexor carpi radialis, i.e. at the supinated wrist and the median nerve was stimulated. Stimuli of 0.2-0.3 $\mathrm{m}$ sec duration were given at 4-7 Hz.

The Intensity of the Current was Adjusted till a Visible Twitch was Produced. Data was Collected and Interpreted as follows:

Filter settings were adjusted to 5-30 Hz. An analysis time of 25-40 msec was chosen to average 1000 sweeps. Measurement was made of peak latency of N20 and P25 (Channel 1), N13 (Channel 2) and N9 (Channel 3). Conduction in other regions were determined using interpeak latency differences like:

1. Conduction through the brachial plexus and cervical cord (Time between N9 at Erb's point and N13 at neck).

2. Conduction from cervical cord and cortex (Time between N13 on the neck and N20 on the scalp).

3. Conduction from the brachial plexus to the cortex (Time between N9 at Erb's point and N20 on the scalp).

The patient was subjected to acupuncture mode of treatment followed by subsequent recording of the SEPs using the same method as mentioned above. Before and after the acupuncture procedures, SEPs were recorded. The data was subjected to appropriate statistical analysis.

\section{Steps for Recording Upper Extremity SEPs were Meticulously followed \\ 1. Display the Select exam and Click on the EP icon. \\ 2. Select SEP under Exam type, then Select the Description "Upper limb 3 Chi w/ Normal averaging." \\ 3. Enter the SEP Record mode. \\ 4. Apply the recording electrodes to both sides; apply the ground and stimulating electrodes to the left side.}


5. Connect the recording and ground electrodes for the left side to the amplifiers and the stimulating electrode to the stimulator box.

6. Check the electrode impedance.

7. Record 2 sets of data from left-side stimulation.

8. Superimpose 2 sets of data from left-side stimulation.

9. Mark and measure responses.

10. Print a screen copy.

11. Store the data in the patient's file.

\section{RESULTS}

Analysis of variance obtained in the data (ANOVA) both pre acupuncture and post acupuncture showed no significant difference in the latencies and inter-peak latencies, but a significant change in N9 and N20 wave amplitudes. (Tables: 1 , $2,3,4,5,6)$.

\begin{tabular}{|c|c|c|c|c|}
\hline Groups & Count & Sum & Average & Variance \\
\hline PRE & 21 & 199.6 & 9.504762 & 1.628476 \\
\hline POST & 21 & 195.8 & 9.32381 & 0.614905 \\
\hline \multicolumn{5}{|c|}{ Table 1 } \\
\hline
\end{tabular}

P value: 0.58 ANOVA: Single Factor N13 Latency

\begin{tabular}{|c|c|c|c|c|}
\hline Groups & Count & Sum & Average & Variance \\
\hline PRE & 21 & 275.8 & 13.13333 & 2.276333 \\
\hline PIOST & 21 & 269.4 & 12.82857 & 0.914143 \\
\hline \multicolumn{5}{|c|}{ Table 2 } \\
\hline
\end{tabular}

P value: 0.43 ANOVA: Single Factor N20 Latency

\begin{tabular}{|c|c|c|c|c|}
\hline Groups & Count & Sum & Average & Variance \\
\hline PRE & 21 & 397.6 & 18.93333 & 3.931333 \\
\hline POST & 21 & 393.1 & 18.71905 & 1.917619 \\
\hline \multicolumn{5}{|c|}{ Table 3 } \\
\hline
\end{tabular}

P value: 0.68 ANOVA: Single Factor N25 Latency

\begin{tabular}{|c|c|c|c|c|}
\hline Groups & Count & Sum & Average & Variance \\
\hline PRE & 21 & -54.894 & -2.614 & 3.605891 \\
\hline POST & 21 & -40.345 & -1.92119 & 3.097632 \\
\hline \multicolumn{5}{|c|}{ Table 4 } \\
\hline
\end{tabular}

P value: 0.22 ANOVA: Single Factor N13 Amplitude

\begin{tabular}{|c|c|c|c|c|}
\hline Groups & Count & Sum & Average & Variance \\
\hline PRE & 21 & $\begin{array}{c}-21.425- \\
1.020238\end{array}$ & & 0.967149 \\
\hline POST & 21 & $\begin{array}{c}-21.49- \\
1.023333\end{array}$ & & 0.562911 \\
\hline \multicolumn{5}{|c|}{ Table 5 } \\
\hline
\end{tabular}

P value: 0.99 ANOVA: Single Factor N20 Amplitude

\begin{tabular}{|c|c|c|c|c|}
\hline Groups & Count & Sum & Average & Variance \\
\hline PRE & 21 & $\begin{array}{c}-14.285- \\
0.680238\end{array}$ & & 1.562401 \\
\hline POST & 21 & $\begin{array}{c}-23.795- \\
1.133095\end{array}$ & & 0.962569 \\
\hline \multicolumn{5}{|c|}{ Table 6 } \\
\hline
\end{tabular}

P value: 0.199 ANOVA: Single Factor P25 Amplitude

\begin{tabular}{|c|c|c|c|c|}
\hline Groups & Count & Sum & Average & Variance \\
\hline PRE & 21 & 35.37 & 1.684286 & 1.392901 \\
\hline POST & 21 & 42.6 & 2.028571 & 3.215575 \\
\hline \multicolumn{5}{|c|}{ Table 7 } \\
\hline
\end{tabular}

P Value: 0.466665 ANOVA: Total: 93.4141141

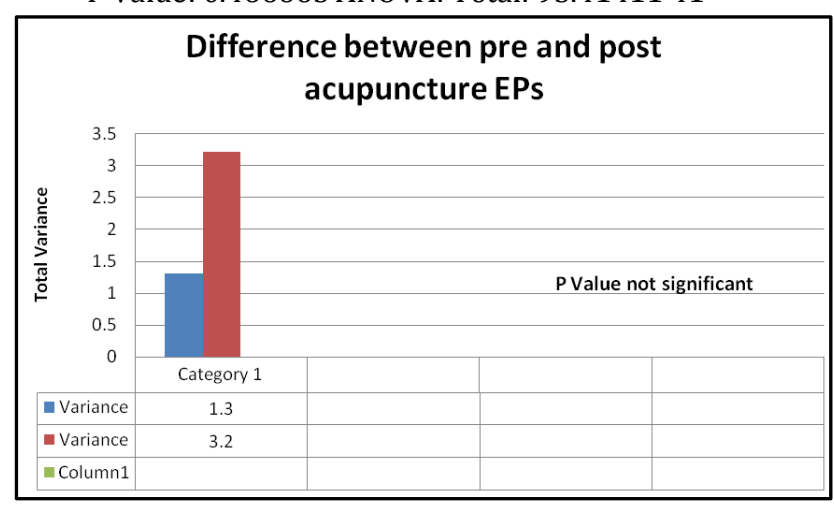

Chart 1

\section{DISCUSSION}

Acupuncture is mainly used as an adjunct to other treatment modalities. Acupuncture is the stimulation of specific acupuncture points along the skin of the body using thin needles. The term Western Medical Acupuncture is used to indicate an adaptation of TCM-based acupuncture, which focuses less on TCM.10 The Western Medical Acupuncture approach involves using acupuncture after a medical diagnosis.

The most common mechanism of stimulation of acupuncture points employs penetration of the skin by thin metal needles, which are manipulated manually or the needle may be further stimulated by electrical stimulation (electroacupuncture). Acupuncture needles are typically made of stainless steel, making them flexible and preventing them from rusting or breaking. Needles are usually disposed of after each use to prevent contamination. ${ }^{11}$ Reusable needles when used should be sterilized between applications. Needles vary in length between 13 to 130 millimetres ( 0.51 to 5.12 in), with shorter needles used near the face and eyes and longer needles in areas with thicker tissues; needle diameters vary from 0.16 $\mathrm{mm}(0.006 \mathrm{in})$ to $0.46 \mathrm{~mm}(0.018 \mathrm{in})$ with thicker needles used on more robust patients. Thinner needles may be flexible and require tubes for insertion. The tip of the needle should not be made too sharp to prevent breakage, although blunt needles cause more pain.

The skin is sterilized and needles are inserted frequently with a plastic guide tube. Needles may be manipulated in various ways including spinning, flicking or moving up and down relative to the skin. Since most pain is felt in the superficial layers of the skin, a quick insertion of the needle is recommended. Often the needles are stimulated by hand in order to cause a dull, localized, aching sensation that is called de qi as well as "needle grasp," a tugging feeling felt by the acupuncturist and generated by a mechanical interaction between the needle and skin.

Off late acupuncture is being increasingly used at academic medical centres, and is usually offered through CAM centres or anaesthesia and pain management services. ${ }^{12}$ Acupuncture has been called 'theatrical placebo.' According to David Gorski when acupuncture proponents advocate 'harnessing of placebo effects' or work on developing 
'meaningful placebos,' they essentially concede it is little more than that. ${ }^{13}$ SLSEPs are widely used in clinical practice, because they are rarely influenced by consciousness and have explicit neural origins. Most studies chose the median nerve, while some stimulated the posterior tibial nerve. The component N20-P25 belonging to the median nerve SLSEP was often observed, because of its definite neural origin (parietal lobe). The components of PREP are all late-latency ones (>150 ms), such as N150, P170-N280 and P260. Electrical (and sometimes magnetic) stimulation on nerves were used to evoke potentials. Modern imaging techniques such as functional magnetic resonance imaging, positron emission tomography, electroencephalography and magnetoencephalography are used to monitor brain activity in humans and may be used to help map the neurophysiological co-relates of acupuncture. The effects of acupuncture on pain perception is controversial. Late amplitudes of somatosensory evoked potentials to noxious stimuli are thought to correlate with the subjective experience of pain intensity. The phenomenon of acupuncture is both complex and dynamic. Acupuncture may exert its actions on pain and immune processes. The coupling of these two systems occurs via common signaling molecules, i.e. opioid peptides. Opioid activation leads to the processing of opioid peptides from their precursor, proenkephalin and the simultaneous release of antibacterial peptides contained within the precursor as well.

Thus, CNS pain circuits may be coupled to immune enhancement. Furthermore, acupuncture needle manipulation signals increase bilaterally in the region of primary and secondary somatosensory cortices in the human brain. Brain maps reveal marked signal decreases bilaterally in multiple limbic and deep gray structures including the nucleus accumbens, amygdala, hypothalamus, hippocampus and ventral tegmental area. A major CNS pathway involvement as well as a local pain and immune modulation activities occur during acupuncture.

Most acupuncture studies have emphasized analgesia and blocking of nociception (the immediate signaling of tissue threat or injury via AS or C fibers). Probably endogenous opioids are involved in this. A neuro-modulating input into the CNS may activate multiple analgesia systems in spinal cord and brain, stimulating the endogenous pain suppression system to release neuro-transmitters (Endogenous opioids).

Separation of the buried EP wave forms from the other electrical activity is accomplished by signal averaging. Stimuli are given repetitively and the computer averages the new data acquired after each stimulus (Random EEG + EP) with the averaged results from previous stimuli stored in its memory. The process is continued until the desired wave forms become sufficiently clarified.

\section{CONCLUSION}

The results of our study suggest that the analgesic effect of Acupuncture does not modify the space and time constants of action potentials conducted along the neuronal pathways to any great extent. Whatever mild effect it has is probably due to modulation of signals at dorsal root ganglia and at the thalamus (Table: 7, Chart: 1). However, more light can be thrown on the subject by examining a larger sample and other nerves.

\section{REFERENCES}

1. Pyne D, Shenker NG. Demystifying acupuncture. Rheumatology 2008;47(8):1132-6. DOI: $10.1093 /$ rheumatology/ken161.

2. Berman BM, Langevin HM, Witt CM, et al. Acupuncture for chronic low back pain. New England Journal of Medicine 2010;363(5):454-61. DOI: $10.1056 /$ NEJMct0806114.

3. White A, Ernst E. A brief history of acupuncture. Rheumatology DOI:10.1093/rheumatology/keg005.

4. Prioreschi P. A history of medicine. Horatius Press, 2004;2:147-8. ISBN 1888456019.

5. Gwei-Djen Lu, Needham J. A history and rationale of acupuncture and moxa. Celestial Lancets 2002.

6. Porter SB. Tidy's Physiotherapy. Churchill Livingstone. Elsevier, 2013:p 403. ISBN 978-07020-4344-4. Retrieved July 14, 2015.

7. Jackson M. The oxford handbook of the history of medicine. Oxford handbooks in history. OUP Oxford. 2011:p 610. ISBN 978-0-19-954649-7. Retrieved July 14, 2015.

8. American Society of Anaesthesiologists Task Force on Chronic Pain Management; American Society of Regional Anaesthesia and Pain Medicine. Practice guidelines for chronic pain management: an updated report by the American society of anaesthesiology task force on chronic pain management and the American society of regional anaesthesia and pain medicine. Anaesthesiology 2010;112(4):810-33.

9. Zeng Y, Liang XC, Yang ZL, et al. Effect of electroacupuncture of hegu (LI 4) on pain-related cerebral somatosensory evoked potentials. Zhen Ci Yan Jiu 2003;28(3):182-8.

10. White A, Cummings M, Filshie J. An introduction to western medical acupuncture. Churchill Livingstone ISBN> 978-0-443-07177, 2008:p 7.

11. William CJ. The American holistic health association complete guide to alternative medicine. New York: Warner Books. ISBN 0-446-67258-0.19.

12. Gorski DH. Integrative oncology: really the best of both worlds? Nature Reviews Cancer 2014;14(10). DOI:10.1038/nrc3822.

13. Colquhoun D, Novella S. Acupuncture is a theatrical placebo: the end of a myth. Anaesthesia \& Analgesia 2013;116(6):1360-3. DOI: 10.1213/ANE.0b013e31828f2d5e. 